\title{
Pulmonary vein isolation in a patient with atrial fibrillation and a filter in the inferior vena cava
}

\author{
Dariusz Rodkiewicz ${ }^{1}$, Marek Kiliszek ${ }^{2}$, Edward Koźluk ${ }^{1}$, Agnieszka Piątkowska ${ }^{1,3}$, Grzegorz Opolski ${ }^{1}$ \\ ${ }^{1} 1^{\text {st }}$ Chair and Department of Cardiology, Medical University of Warsaw, Warsaw, Poland \\ 2Department of Cardiology and Internal Diseases, Military Institute of Medicine, Warsaw, Poland \\ ${ }^{3}$ Chair of Emergency Medicine, Wroclaw Medical University, Wroclaw, Poland
}

Adv Interv Cardiol 2017; 13, 3 (49): 279-280

DOI: https://doi.org/10.5114/aic.2017.70205

A 60-year-old man with a 1-year history of highly symptomatic drug-resistant paroxysmal atrial fibrillation (AF) was admitted for pulmonary vein isolation (PVI). In 1984 the patient had hemorrhagic stroke of the left hemisphere with right-sided hemiparesis that was complicated with pulmonary embolism. Therefore the patient was treated with surgical implantation of a prototype filter to the vena cava inferior ( $\mathrm{VCl}$ ). His medical history included hypertension, diabetes mellitus, transient ischemic attack and epilepsy.

Using the transfemoral approach under fluoroscopic guidance, the diagnostic catheter was placed in the coronary sinus. Crossing the filter with the long guidewire of the transseptal sheath (8.5 Fr SLO, St Jude Medical, Minnetonka, MN, USA) turned out to be impossible. To overcome this problem we advanced the transseptal sheath over the guidewire next to the filter and only then managed to pass the guidewire (and then the sheath) by the filter (Figure 1). Double transseptal puncture was performed successfully. Using the CARTO system, Lasso diagnostic catheter (Biosense Webster, Diamond Bar, Ca, USA) and Navistar ThermoCool Smarttouch ablation catheter (Biosense Webster, Diamond Bar, Ca, USA), successful PVI was performed. The overall procedure time was $215 \mathrm{~min}$. Total fluoroscopy exposure was $31 \mathrm{~min}$ and $53 \mathrm{~s}\left(6141.9 \mathrm{cGy} \cdot \mathrm{cm}^{2}\right)$. During 12 months of follow-up there was no AF (24-hour ECG monitoring at 3, 6, 9, and 12 months after ablation).

The $\mathrm{VCl}$ filters are inserted for prevention of pulmonary embolus from lower extremity deep vein thrombosis [1]. Presence of a VCI filter has traditionally been considered a relative contraindication for catheterization from the femoral vein [2]. To our knowledge, this is the first case of successful PVI in a patient with $\mathrm{AF}$ and presence of an unknown type of filter in the $\mathrm{VCl}$. The decision to perform the catheter procedure largely depends on the filter type in the $\mathrm{VCl}$. Over the past decade, there has been a gradual evolution of $\mathrm{VCl}$ filters [3]. Limited data are available on complicating the standard procedure catheterization across the filter in the $\mathrm{VCl}$ [4]. During crossing the filter we used continuous fluoroscopy to reduce the risk of adverse events. It was necessary to bring the sheath close to the filter. The solution was to use a straight guidewire for passage of the filter. All maneuvers were performed without filter dislodgment. The PVI is possible but much more complicated in patients with a filter in the $\mathrm{VCl}$.

\section{Conflict of interest}

The authors declare no conflict of interest. 

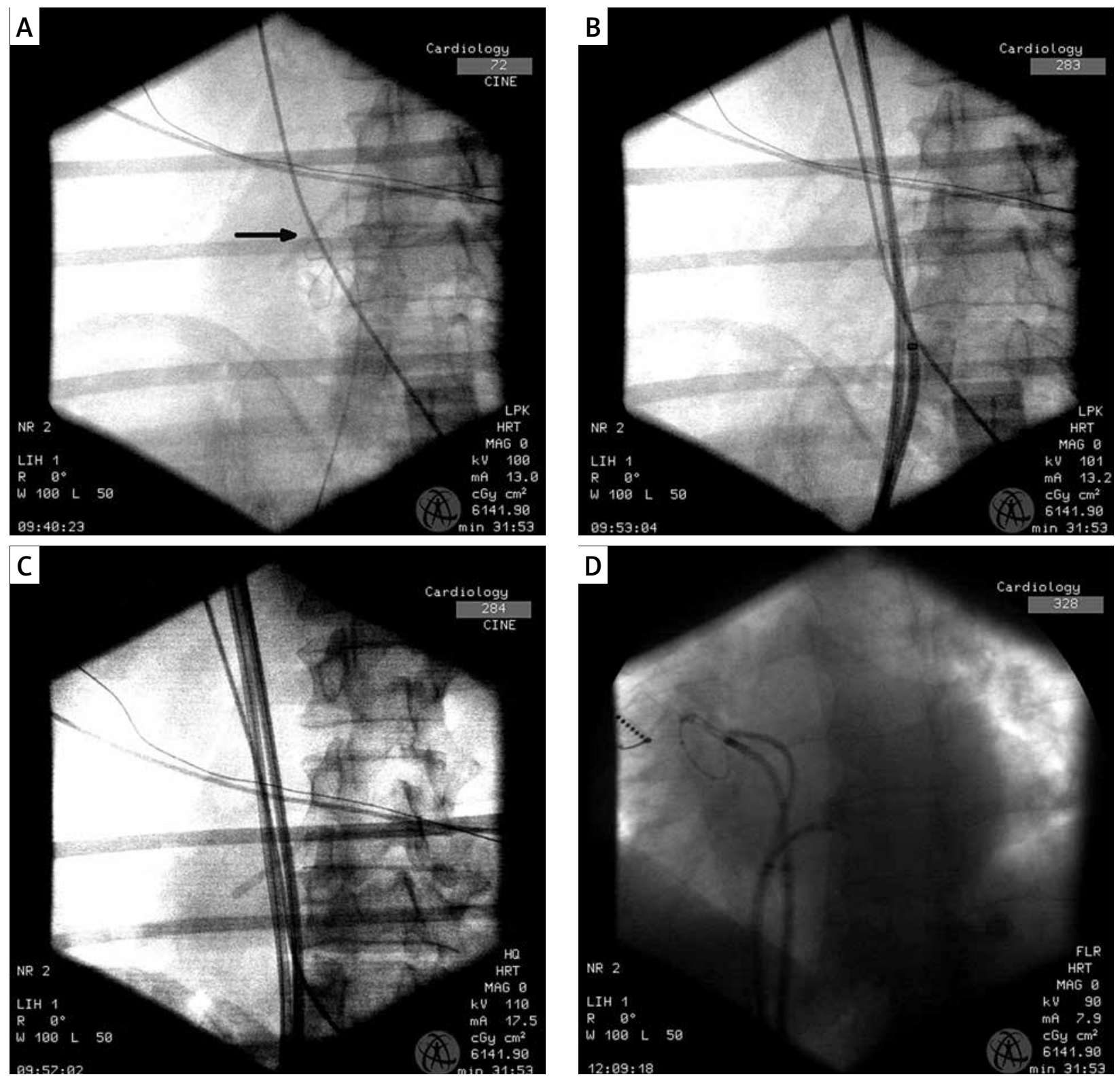

Figure 1. A - Fluoroscopic AP view. Difficulties with crossing the filter (black arrow) in the vena cava inferior with the guidewire. The guidewire was twisted in a pigtail beneath the filter. The diagnostic steerable catheter was successfully passed through the filter. B - Fluoroscopic AP view. We repositioned the transseptal sheath over the guidewire next to the filter. This maneuver allowed passage through the filter by the guidewire and then the transseptal sheath. C - Fluoroscopic AP view. Both transseptal sheaths and the diagnostic catheter were successfully passed through the filter. D - Fluoroscopic AP view. Ablation catheter and lasso catheter in the ostium of the right superior pulmonary vein during pulmonary vein isolation

\section{References}

1. Schoeffler M, Ringewald J, Schechter E. Transfemoral venous access through inferior vena cava filters for interventions requiring large sheaths. Catheter Cardiovasc Interv 2007; 69: 47-51.

2. Sinha SK, Harnick D, Gomes JA, Mehta D. Electrophysiologic interventions in patients with inferior vena cava filters: safety and efficacy of the transfemoral approach. Heart Rhythm 2005; 2: $15-8$
3. Kalva SP, Chlapoutaki C, Wicky S, et al. Suprarenal inferior vena cava filters: a 20-year single-center experience. J Vasc Interv Radiol 2008; 19: 1041-7.

4. Kanjwal Y, John JM, Burket MW. Passing sheaths and electrode catheters through inferior vena cava filters: safer than we think? Catheter Cardiovasc Interv 2009; 74: 966-9. 